\title{
Adaptive Bandwidth Allocation for QoS Guaranteed VLC Based WPAN
}

\author{
Nirzhar Saha*, Ratan Kumar Mondal*, Yeong Min Jang
}

\begin{abstract}
Visible light communication based wireless personal area network (WPAN) has recently remained in the center of attention, as it shows a lot of promise to a be a perfect replacement for the contemporary Radio frequency (RF) communication at least in the indoor environment. A commercially deployable VLC based WPAN must support diverse traffic requirement for different kinds of service. In this paper, we have proposed an innovative bandwidth allocation scheme for VLC based WPAN. We wish to allocate bandwidth adaptively for users in a network where bandwidth for each user is allocated scalably. Our aim is to allow maximum number of users in a VLC based WPAN where each user is guaranteed their required QoS. The simulation results justify that the proposed scheme is better than the conventional scheme.
\end{abstract}

Key Words : VPAN, adaptive bandwidth allocation, QoS.

\section{I . Introduction}

Wireless personal area networks (WPAN) is a fast growing technology with a focus on enabling the wireless sensor networks (WSN). It attempts to provide a scalable data rate, low power, and low cost wireless networking on the device-level communication. There is one limitation to WPAN which hampers widespread use of this technology in indoor communication. The data rate offered by the state of the art WPAN is fairly limited. Therefore research is moving towards the direction of improving the data rate. Wireless links under 802.15.4 can operate in three license free industrial scientific medical (ISM) frequency bands which accommodate over air data rates of $250 \mathrm{kbps}$ in the $2.4 \mathrm{GHz}$ band, $40 \mathrm{kbps}$ in the $915 \mathrm{MHz}$ band, and $20 \mathrm{kbps}$ in the $868 \mathrm{MHz}^{[1-2]}$. On the other hand, next generation wireless application such as smart phones and smart televisions and other devices target services based on multimedia content. There is a significant demand for applications such as high definition (HD) video streaming, gaming, video conferencing etc. Now a days a lots of multimedia contents are shared in the internet and it's increasing in a rapid pace. Therefore current WPAN is unable to satisfy the bandwidth demand of the users. On the other hand, spectrum i.e. bandwidth is a scarce resource. Either borrowing spectrum or expanding the range of spectrum to provide high data rate will add more to the RF spectrum shortage.

To provide improved data rate a new communication network has been proposed in the IEEE standard 802.15.7 [3] using visible light medium. This standard describes the use of visible light communication (VLC) for WPAN merging data

\footnotetext{
※ This work was supported by the IT R\&D program of MKE/KEIT [10035362, Development of Home Network Technology based on LED-ID].

- 주저자 : Department of Electronics Engineering, Kookmin University, nirzharsaha_eee@yahoo.com, 학생회원

- 교신저자 : Department of Electronics Engineering, Kookmin University, yjang @kookmin.ac.kr, 종신회원

* Department of Electronics Engineering, Kookmin University

논문번호 : KICS2013-06-252, 접수일자 : 2013년 6월 15일, 최종논문접수일자 : 2013년 8월 9일
} 
communication and lighting application together, in order to deliver data rates sufficient enough to support audio and video multimedia services. It is capable of utilizing the unlicensed visible spectrum between $380 \mathrm{~nm}$ to $780 \mathrm{~nm}$ in wavelength. Therefore, devices in a VLC based WPAN which is also known as visible light personal area network (VPAN). VPAN is an optical wireless communication system using light emitting diodes (LEDs) as a transmitters and photo sensitive receivers. Photo diodes (PDs) are used to make photo sensitive receivers. It can provide ubiquitous ultrahigh speed wireless communication in any indoor environment with less interference. Till now the research work on VLC based WPAN is limited to physical and medium access control (MAC) layer designing. Therefore, it is high time to focus on quality of service (QoS). Like any other wireless communication system, QoS provisioning is a crucial issue in VLC based WPAN.

In this paper, we concentrate on the adaptive resource allocation for the users in VLC based WPAN. From the study, it is understandable that each type of user has different kinds of quality of service (QoS) requirement. For example, the device used for cellular communication requires much less bandwidth compared with another user uses video streaming service. Specially for realtime voice and video application a minimum rate must be guaranteed for each user. Otherwise the users of those application may suffer outage. Therefore it is important to allocate bandwidth efficiently so that the system can serve as many users as possible. However it is practically impossible to satisfy the rate/capacity of each user in the system. Therefore we propose an adaptive bandwidth allocation scheme to allocate bandwidth effectively among users. The proposed algorithm aims to allocate scalable bandwidth to each user with guaranteed QoS and at the same time admit maximum possible number of users in the system. We present formulation for bandwidth allocation by using linear optimization scheme. Then a greedy search scheduler is developed in order to reduce computational complexity in case of admitting new users.
The rest of the paper is structured as follows. We present a synopsis over VLC based WPAN system and channel model in Section II. In Section III, we present our resource allocation scheme regarding bandwidth allocation. Section IV presents numerical result, followed by conclusion in section $\mathrm{V}$.

\section{VLC Based WPAN: An Introduction}

In this section, we are going to give an overview on simple VLC based WPAN which has been subjected to vigorous study by many researchers in the past few years as an understudy of contemporary WPAN. In a VLC based WPAN, visible light is used as a transmission medium for communication among the transmitters and receivers. Optical wireless communication technology by using either visible light or infrared spectrum is envisioned to as the next generation indoor wireless communication system. The transmitters are composed of high brightness light emitting diodes (LED) in typical VLC based WPAN of which light intensity is modulated according to the data to be transmitted. Light signals from LED sources can be intensity modulated at very high-speed. The optical signal produced in this process is easy to detect. The intensity modulated signal is easily recovered from the output of a photo diode (PD). Because of the high energy efficiency of the LED, it is possible to achieve high optical signal to noise ratio using only a few watts of power. This type of modulation and detection process is generally known as intensity modulation-direct detection (IM-DD). The IM-DD scheme suffers from couple of disadvantages which are reduction in sensitivity, and noise vulnerability. It is also affected by signal over lapping problem in the PD when the transmitter sends different signal for different users even though it is relatively easy to implement compared to other wireless schemes. When users require information provided by the data server via transmitters. Coordinator which actually is a controller, controls the over all data communication procedure. It requires two stages for data 
communication $^{[4]}$. In the pre-identification stage an user is identified by transmitter and then user sends a request for desired information. In the post-identification stage, based on the user's request, transmitter will communicate by a channel allocated by the coordinator.

\subsection{Channel Model}

Consider an optical wireless communication network using VLC based WPAN. The whole coverage area inside a VLC based WPAN is divided into $M$ cells. There are total $N$ users. Each cell has $K$ number of channels. A spectrum of total bandwidth $W_{t}$ is available for the transmissions from the sources. This spectrum is assumed to be slow/shadow fading and can be divided into distinct and non-overlapping channels of equal or unequal bandwidths. Depending on the application the coordinator can decide how to allocate bandwidth. A VLC based WPAN uses visible light spectra. Modulated optical signal by transmitter is received by using an optical receiver. The quality of received optical signal depends on optical channel gain and transmitted signal power. The received optical signal can be expressed as ${ }^{[5]}$

$$
P_{r}=R_{P D} H(0) P_{t}+n_{t}
$$

where, $R_{P D}$ is the responsivity of the PD. $P_{r}, P_{t}$ are the received and transmitted power respectively. $H(0)$ is the optical channel gain. $n_{t}$ is the additive white Gaussian noise which is dominated by shot noise of the PD. Now the SINR equation for user $n$ can be expressed as

$$
\gamma_{n}=\frac{R_{P D} H_{n} P_{n}}{\sum_{n=1}^{N-1} R_{P D} H_{n} P_{n}+\xi}
$$

where $H_{n}$ is the optical channel gain for channel k. $P_{n}$ is the transmitted power. We assume the transmitted power is constant for all users (/in every cell). $\xi$ is the cumulative noise power. Now we need to calculate the channel capacity. The maximum data rate at which reliable communication is possible is called the capacity of the channel. Now the Shannon's formula for AWGN channel can be written as

$$
R_{n}=W_{n} \log _{2}\left(1+\frac{\gamma_{n}}{W_{n}}\right)
$$

Here, $R_{n}$ is the channel capacity and $W_{n}$ is the assigned bandwidth for $n^{\text {th }}$ user respectively.

\section{Resource allocation in VLC based WPAN}

A radio resource management (RRM) framework is required for a wireless network to achieve the desired network objective under the constraint on available radio resources (e.g. the bandwidth or time slot, or transmission power). The efficient allocation of available radio resource for multiuser VPAN remains an open issue since very little attention has been given to allocate resource efficiently. The primary objective of using VLC based WPAN is the possibility of providing extra amount of bandwidth to support all kinds of device in the indoor environment over its RF counterpart. Ideally LED enabled WPAN has bandwidth in $\mathrm{THz}$ range, but recent study has ${ }^{[6]}$ unveiled that the effective bandwidth of the VLC based WPAN is restricted by the frontend design of the transmitter and receiver. Therefore resource allocation e.g. bandwidth allocation requires special attention.

Like other wireless multi-user networks it is very important to allocate available radio resource efficiently in VLC based WPAN. There are several schemes for bandwidth allocation, depending on the application and the deployment scenario. In some cases, power allocation is considered jointly with bandwidth allocation in order to boost the capacity for each user. In the ${ }^{[7]}$ joint power allocation with admission control has been proposed in VLC based WPAN. Time/bandwidth allocation strategies with constant transmit power have been developed based on the time division multiple access/frequency 
division multiple access (TDMA/FDMA) to optimize effective capacity in relay networks ${ }^{[8]}$. The most commonly used approaches use fixed bandwidth allocation. The problem related to equal bandwidth allocation is that some services may not use the all the allocated bandwidth. Therefore, some portion of the bandwidth in the system will remain unused even though another services may require extra amount of bandwidth to maintain its' QoS. The idea is to use this unused bandwidth for another service which requires more bandwidth and thus maintain QoS for that service. The allocation of bandwidth must be scalable and adaptive therefore bandwidth utilization of the system will be enhanced from the existing VLC based WPAN. In fact, it is not efficient to allocate the bandwidth equally when the total available bandwidth is limited. In some applications, certain minimum transmission rates must be guaranteed for the users in order to satisfy their quality-of-service (QoS) requirements. For example, in real-time voice and video applications, a minimum rate should be guaranteed for each user to satisfy the delay constraints of the services. However, when the rate/capacity requirements can not be supported for all the users, admission control is adopted to decide which users to be admitted into the network.

\subsection{Equal and Fixed Bandwidth Allocation}

The problem of equal bandwidth allocation can be explained from the Shannon's channel capacity equation. Now for equal allocation the overall bandwidth $W_{t}$ is divided by $N$ users. Therefore allocated bandwidth to each user can be expressed as $W_{\text {alloc }}=\frac{W_{t}}{N}$. Here the number of users, $N$ those can be admitted into the network is limited to the QoS requirement.

$$
R_{\text {equal }}=\frac{W_{t}}{N} \log _{2}\left(1+\frac{\gamma_{k}}{W_{t} / N}\right)
$$

From the above equation it is clear that capacity depends on several constraints which are bandwidth, SINR, and several physical properties of the transmitter and receiver, specially for VLC based WPAN. Distance between transmitter and receiver nodes, field of view (FOV) and effective area of the PD include such physical properties. Therefore when SINR changes, channel capacity/ highest data rate will change and results QoS degradation. The main problem with fixed and equal allocation is that it results inefficient allocation even though all types of services might not require same amount of bandwidth. In other words this type of allocation results bandwidth wastage.

\subsection{Adaptive Bandwidth Allocation}

Considering multiuser enabled wireless network system should support scalable data rate to satisfy each user QoS. For multiuser resource allocation under QoS constraint, a scalable control mechanism is needed. By using linear optimization algorithm it is possible to allocate scalably where users are allocated different amount of bandwidth by coordinator as, $\mathrm{W}^{*}$ $=\left(\mathrm{W}_{1}, \mathrm{~W}_{2}, \cdots, \mathrm{W}_{\mathrm{K}}\right)$ on each channel. Thus, the formulation for bandwidth allocation takes the form

Maximize

$$
R(W)=\sum_{n=1}^{N} \frac{W_{n}}{N} \log _{2}\left(1+\frac{N \gamma_{n}}{W_{n}}\right)
$$

s.t.

$$
\begin{aligned}
& \sum_{n=1}^{N} W_{n} \leq W_{\text {available }} \\
& R_{i} \geq \lambda_{i}^{q} \quad i \in N
\end{aligned}
$$

The objective function in equation (5) aims to maximize capacity of each user. The constraint in equation (6) allocate bandwidth for new user where as constraint in (7) ensures that the user QoS is satisfied. $\lambda_{i}^{q}$ is the rate constraint in (7). Note that $W_{\text {available }} \leq W_{t}$. The optimization scheme presented in (5), (6), and (7) has one non-negative constraint. By transforming equation (5) into a minimization problem and also transforming (7) into negative constraint, the set of equation can be made to convex one ${ }^{[9]}$. the by using the Lagarangian approximation we can find the optimized bandwidth for each channel. 
Table 1. Simulation parameters

\begin{tabular}{|l|c|}
\hline \multicolumn{1}{|c|}{ Parameter } & Value \\
\hline Transmitted optical power & $1 \mathrm{~W}$ \\
\hline System bandwidth & $10 \mathrm{MHz}$ \\
\hline SINR threshold & $3 \mathrm{mB}$ \\
\hline Distance between reader and tag & $150 \mathrm{~mm}^{2}$ \\
\hline Physical area of the PD & 1.0 \\
\hline Transmission coefficient of filter & 60 (degree) \\
\hline Concentrator FOV & 15 (degree) \\
\hline Semi-angle at half power & 1.5 \\
\hline Refractive index & 10 \\
\hline Number of users & \\
\hline
\end{tabular}

\section{Performance Analysis}

According to our proposed model, we performed a numerical simulation for equal and adaptive bandwidth allocation scenario. The values of the parameters are listed in table 1 , which we used in our simulations. We keep the SINR level constant at the value of $10 \mathrm{~dB}$ and the number of admitted users under a single transmitter is restricted to 10 . We performed numerical simulation by using MATLAB. We observed that adaptive bandwidth allocation scheme achieve better performance comparing with equal bandwidth allocation scheme at constant SINR and user, shown in fig. 1.

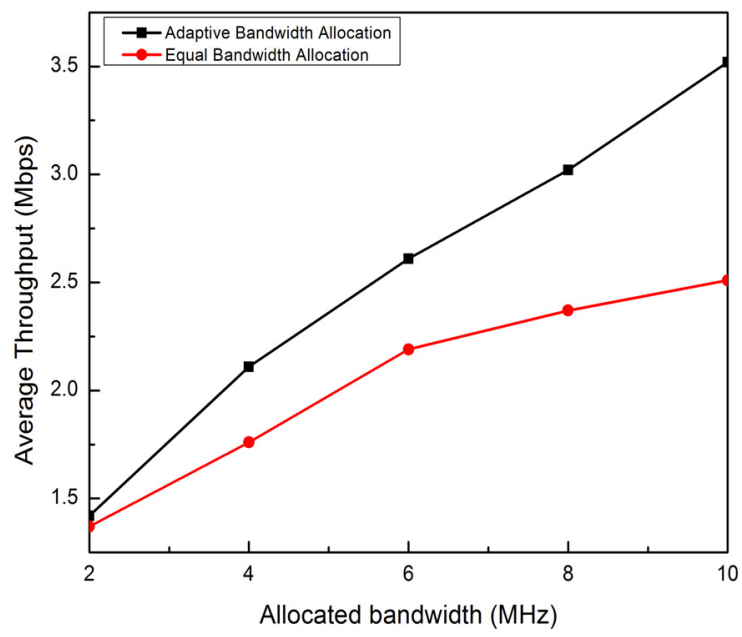

Fig. 1. Allocated bandwidth vs average throughput.

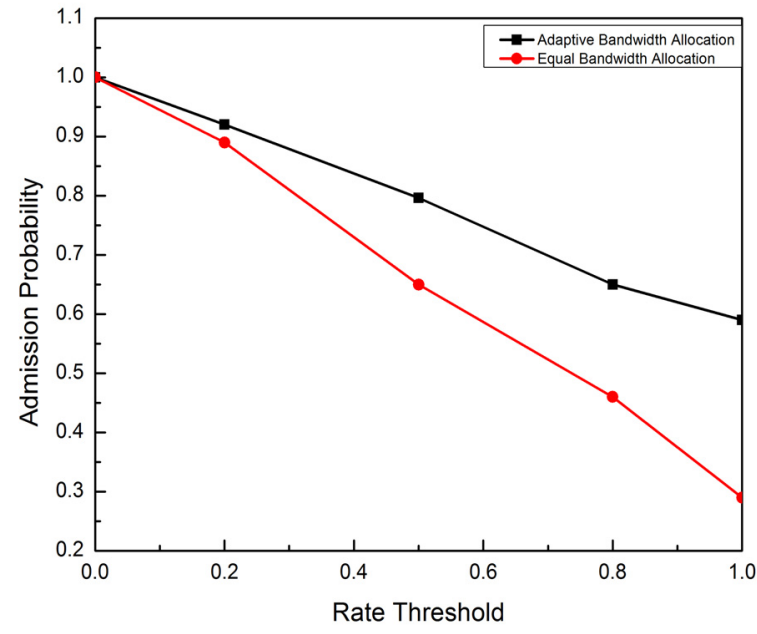

Fig. 2. Admission probability vs Rate threshold.

It is observed that adaptive bandwidth allocation can guarantee up to $28.6 \%$ boost in average throughput. Admission probability versus rate threshold is shown in fig. 2. The number of admitted user under a transmitter is restricted by rate threshold. Which means user has to provide with its' rate (QoS). From fig. 2 it is found that adaptive bandwidth allocation outperforms fixed bandwidth allocation. It is found that admission probability of adaptive bandwidth allocation scheme is about 30\% more than equal bandwidth allocation schemes.

\section{Conclusions}

VLC based WPAN is going to be the new paradigm in a quest for green and high speed communication. However resource allocation (bandwidth, power allocation) remains a challenging issue specially in case of commercial deployment. In order to provide scalable high data communication an adaptive bandwidth allocation has been proposed for VLC based WPAN. The proposed scheme can allocate bandwidth adaptively and scalably and can admit more users comparing with conventional equal bandwidth allocation. The proposed scheme is also able to provide higher percentage of throughput. Simulation result shows significant improvement in performance. 


\section{References}

[1] J. S. Lee, "Performance evaluation of IEEE 802.15.4 for low-rate wireless personal area networks," IEEE Trans. Consum. Electron., vol. 52 no. 3, pp. 742-749, Aug. 2006.

[2] E. Hossain, D. Niyato, and Z.Han, Dynamic Spectrum Access and Management in Cognitive Radio Networks, Cambridge University Press, 2009.

[3] IEEE, IEEE Standard for Local and Metropolitan Area Networks, Part 15.7: Short-Range Wireless Optical Communication Using Visible Light, IEEE Std. 802.15.7-2011, Sep. 2011.

[4] V. V. Huynh, N. T. Le, S. Choi, and Y. M. Jang, "Collision reduction using modified Q-Algorithm with moving readers in LED-ID system," J. KICS, vol. 37A, no. 5, pp 358-366, May 2012.

[5] T. Komaine, and M. Nakagawa, "Fundamental analysis for visible-light communication system using LED Lights," IEEE Trans. Consum. Electron., vol. 50, no. 1, pp. 100-107, Feb. 2004.

[6] B. Ghimirie, and H. Hass, "Self-organising interference coordination in optical wireless networks," EURASIP J. Wireless Commun. Networking, vol. 2012, Article no. 131, Apr. 2012.

[7] N. Saha, R. K. Mondal, and Y. M. Jang, "Opportunistic channel reuse for a self-organized visible light communication personal area network," in CRCN(Cognitive Radio Commun. Networks) Session, in Int. Conf. Ubiquitous Future Newtorks (ICUFN), Session 3A-2, July 2013.

[8] L. Xie and X. Zhang, "TDMA and FDMA based resource allocations for quality of service provisioning over wireless relay networks," in Proc. IEEE Wireless Commun. Networking Conf. (WCNC 2007), pp. 3153-3157, Kowloon, China, Mar. 2007.

[9] T. H. Cormen, C. E. Leiserson, and R, L. Rivest, Introduction to Algorithms, $3^{\text {rd }}$ Ed., MIT Press, 2009.
Nirzhar Saha

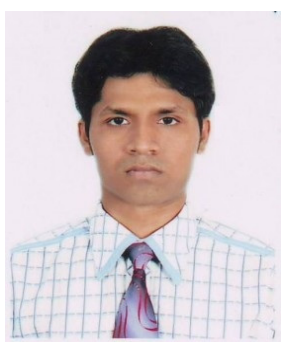

$\mathrm{He}$ received his B.Sc. in Electrical and Electronic Engineering from Khulna University of Engineering and Technology, Bangladesh in 2011. In 2012, he joined the wireless networks and communication lab as a research student in the department of Electronics Engineering at Kookmin University, Korea. His research interests include visible light communication networks, QoS management, cognitive radio networks.

Ratan Kumar Mondal

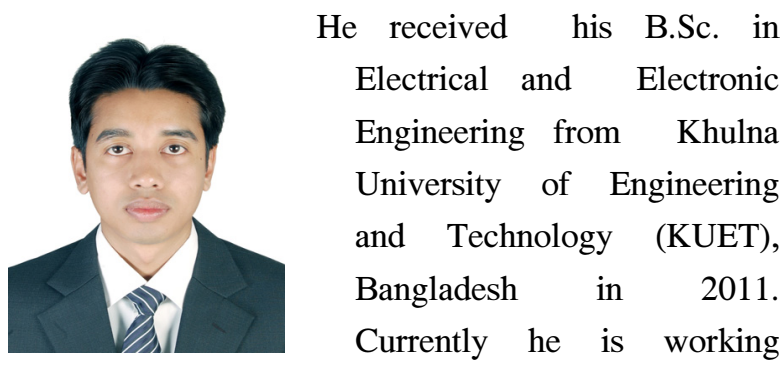
towards his M.Sc. degree in the department of Electronics Engineering at the Kookmin University. His research interests include visible light communication, localization, and resource management.

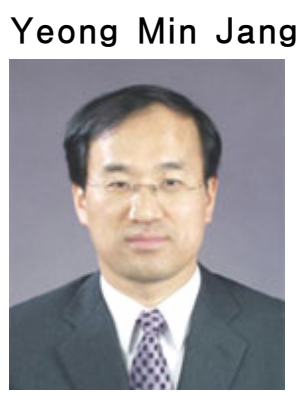

$\mathrm{He}$ received the B.E. and M.E. degree in Electronics Engineering from Kyungpook National University, Korea, in 1985 and 1987, respectively. $\mathrm{He}$ received the doctoral degree in Computer Science from the University of Massachusetts, USA, in 1999. He worked for ETRI between 1987 and 2000. Since September 2002, he is with the School of Electrical Engineering, Kookmin University, Seoul, Korea. His research interests include $\mathrm{B} 4 \mathrm{G}$, radio resource management, femtocell networks, Multi-screen convergence services, and WPANs. 